\title{
Use of Medicines Changing the Face of Abortion
}

\section{By Beverly Winikoff \\ and}

Wendy Sheldon

Beverly Winikoff is president and Wendy Sheldon is senior program associate, both at Gynuity Health Projects, New York.
Medication abortion (also commonly referred to as medical abortion) is arguably the most important advance in reproductive health technology since the discovery of oral contraceptives. This simple process, involving the use of pills rather than invasive surgical instruments, has been shown to be safe and up to $98 \%$ effective for early pregnancy termination. ${ }^{1-3}$ In settings where abortion is legal, medication abortion has expanded the array of effective options available, thus improving the abortion experience for those who wish to avoid surgery. In settings where abortion is illegal or highly restricted, it has provided many women for the first time with a safe and discreet means for early termination of unwanted pregnancy. Further, in all settings where it is available, medication abortion has reduced women's dependence on medical systems, providing them with greater autonomy and control over their most important reproductive decisions.

Administration of mifepristone, followed by a prostaglandin, is the gold standard for medication abortion. Mifepristone was initially approved in the late 1980s in China and France and has since been safely and effectively used by many millions of women worldwide. ${ }^{4-7}$ Mifepristone diminishes the biological availability of progesterone, the hormone needed to sustain pregnancy. It is typically used in combination with misoprostol, which helps empty the uterus by inducing uterine contractions. ${ }^{1}$ However, mifepristone is not available in many settings because of legal restrictions on the provision of abortion services. It is also unavailable in numerous settings where the population of reproductive-age women is small, since the high cost of drug registration makes entering the market unappealing to commercial entities. As a result, mifepristone is registered for pregnancy termination in only about 50 of the world's countries, the majority of which are in the developed regions of Europe. ${ }^{8}$ Of the 20 developing countries where mifepristone is registered, two-thirds are in Asia. At present, mifepristone is registered in just five African countries (Mozambique, Zambia, Tunisia, South Africa and Ghana) and two Latin American countries (Mexico and Guyana).

In many settings where mifepristone is unavailable, misoprostol is commonly used alone for early pregnancy termination. Misoprostol was originally developed in the mid-1980s for gastrointestinal indications and is licensed for such purposes in about 90 countries worldwide. ${ }^{9,10}$
The drug is inexpensive and stable at room temperature, making it ideal for inducing abortion in low-resource settings. The first reported widespread use of misoprostol for pregnancy termination was in Brazil in the late 1980s. ${ }^{11,12}$ Since then, knowledge and use of misoprostol by itself as a means of early pregnancy termination have grown throughout Latin America, as well as in other regions where abortion is legally restricted and mifepristone is unavailable. ${ }^{13,14}$ Although use of misoprostol alone is less effective than its use with mifepristone, misoprostol-only abortions are nevertheless a safe, acceptable and effective means of early pregnancy termination. ${ }^{6,15,16}$ In still other settings, women lack access to both misoprostol and mifepristone. These include much of northern and western Africa; some parts of middle, eastern and southern Africa; and a number of countries in Asia and South America. ${ }^{10}$ Perhaps not surprisingly, some of the world's most restrictive abortion laws and highest rates of abortion-related mortality are found in these same places. ${ }^{17,18}$

The acceptability of medication abortion among women seeking an abortion is high in developing countries, regardless of the setting or regimen used., ${ }^{5,6,19}$ Furthermore, demand for the procedure appears to be increasing rapidly in many places. It is difficult to document specific trends in the use of medication abortion, because mifepristone is frequently unavailable and use of misoprostol is largely offlabel or clandestine. There is nevertheless some evidence of recent growth in sales of both products. In one study that tracked sales of misoprostol between 2002 and 2007, there were substantial increases in a number of Asian countries, including India (646\%), Bangladesh (128\%) and Indonesia (118\%), and regional increases of $86 \%$ in the Middle East-North Africa and 27\% in Sub-Saharan Africa. ${ }^{20}$ Other evidence also suggests large increases in recent demand for medication abortion in India, where mifepristone and misoprostol are both registered and widely available. According to pharmaceutical sources, 100 million tablets of misoprostol were sold in 2011 (up from 34 million in 2007). ${ }^{21}$ Although comparable data from earlier years are not available for mifepristone, approximately 20 million tablets of the drug were sold in the country in $2011 .^{21}$

Growth in the use of medication abortion in developing countries is likely to continue unabated because population growth ensures that the number of women of repro- 
ductive age will increase, and because knowledge of the procedure is becoming widespread. For example, in places where women have little or no access to either drug, some are turning to the Internet for purchase of the medications. Women on Web, which was established in 2006 to help women from countries with restrictive abortion laws, is one provider of such services. After confirming the client's eligibility online, Women on Web mails a package containing mifepristone, misoprostol and a pregnancy test kit to the woman's home. Women on Web has served thousands of women from at least 88 countries in Western and Eastern Europe, the Middle East, Africa, Asia and Oceania, and Latin America and the Caribbean. ${ }^{22}$ Evidence suggests that the use of telemedicine by Women on Web and other medical professionals can be a safe and effective means for providing medication abortion. ${ }^{23,24}$ On the other hand, Internet-based services cannot reach those without access to computers, or many who reside in rural areas.

Another factor responsible for the rising use of medication abortion in some developing country settings is increased involvement of midlevel providers in provision of the procedure. For example, nonphysician clinicians are either providing or have been found able to provide medication abortion in Vietnam, Tunisia, South Africa and Nepal. ${ }^{25-28}$ Evidence suggests that provision by nonphysician clinicians has no adverse impact on the safety or efficacy of the procedure. ${ }^{25,28}$ In addition, new ways of simplifying services and reducing dependence on the medical system have been developed. For example, a new pregnancy test now under development would make it feasible for women to track changes in their levels of human chorionic gonadotropin (hCG) over time.* The test is suitable for at-home use following medication abortion and has the potential to reduce substantially the need for in-clinic follow-up visits. In the United States and Vietnam, the test has been shown to be highly effective at diagnosing ongoing pregnancy following medication abortion, and the majority of women in both settings reported that the test was easy to administer. ${ }^{29,30}$

Yet, there are still important barriers that limit access to medication abortion in developing countries. Although legal reform is essential for provision of overt services in settings where abortion is illegal or highly restricted, there are also barriers in places where abortion is legal and accepted. Political and regulatory choices at all levels of the health care system can have a pronounced impact on the cost and availability of the drugs used for medication abortion.

For example, there may be good availability of mifepristone or misoprostol in the private sector but insufficient access to medication abortion in the public sector. This is the current situation in India, where abortion is legal through 20 weeks of pregnancy, and mifepristone and misoprostol were approved for sale and manufacture throughout the country in 2002. Following approval, the number of medication abortion products available in India for purchase in the private sector rapidly expanded and sales subsequently boomed. By 2009, at least 20 generic misoprostol prod- ucts were available in the country in varying formulations, and at least seven companies were marketing a combination pack for medication abortion that included one tablet of mifepristone and four tablets of misoprostol (200 mcg each). However, medication abortion is still largely unavailable in public-sector services in most states, and health care providers often lack sufficient knowledge about the use and advantages of medication abortion. ${ }^{31}$

In other settings, the public sector serves as the main provider of medication abortion and the private sector plays a very small role. This is largely the situation in Mexico City, where elective abortion was legalized through 12 weeks since the last menstrual period in 2007. Within months of the law's passage, the Mexico City government had agreed on a misoprostol regimen and incorporated it into clinical guidelines for their public hospitals and health care centers, where the procedure is free of charge to Mexico City residents. In the period between 2007 and March 2012, public-sector facilities provided a total of 78,788 abortions, 51,747 (66\%) of which were induced with medication. ${ }^{32}$ Mifepristone was registered in Mexico in 2011 and has since been incorporated into public-sector abortion services. ${ }^{8}$ Use of medication abortion has continued to increase among women who receive abortions; in the first three months of 2012 medication abortion accounted for $75 \%$ of all abortion procedures provided. ${ }^{32}$ In contrast with the situation in India, the vast majority of medication abortions in Mexico are provided through government channels, rather than the private or nongovernmental sectors.

In other places, such as parts of the former Soviet Union, the use of medication abortion is hindered by unnecessary requirements for women or health care systems. In Kazakhstan, for example, use of medication abortion is low, despite the availability of the drugs, because of requirements that women who obtain the procedure must stay in the hospital, obtain STI testing and return for a follow-up visit. In Uzbekistan and the Southern Caucasus (Armenia, Azerbaijan and Georgia), cumbersome drug registration requirements mandate re-registration of mifepristone and misoprostol every five years, and medication abortions cannot be provided until re-registration is completed. ${ }^{33}$ In addition, informal costs that ultimately drive up the price of the procedure are common, frequently as a result of corruption or inefficiency in the commercial sector. For instance, in Ukraine one must purchase a 600-mg dose of mifepristone, although a dose of $200 \mathrm{mg}$ is the standard regimen recommended in national guidelines by the Ministry of Health. Because mifepristone is a relatively expensive drug (three pills cost about US\$270), the required purchase of excess medication adds substantially to the cost of the abortion.

Although access to medication abortion is still limited in many places, there has been considerable progress in a

*Normal pregnancy tests provide information only about whether any hCG is detectable. 
very short time. Awareness of the use of drugs to induce abortion now exists almost everywhere; demand has transcended cultural, educational and geographic boundaries; and women from around the world are consistently satisfied with the procedure. ${ }^{19}$ If we are to achieve universal access, however, we must find ways to introduce the technology wherever it is currently unavailable. Medication abortion-like surgical abortion-is being used in countries where abortion is illegal or highly restricted and will continue to be sought there in the future. In such settings, medication abortion is likely to be safer, less invasive, and more acceptable than surgical abortion. Finally, all women who seek medication abortion should have access to the combination of mifepristone and misoprostol, the regimen with the highest efficacy and the fewest side effects. Medication abortion is now an indispensable part of abortion care in developed countries. Equitable access for women in developing countries is the next imperative.

\section{REFERENCES}

1. World Health Organization (WHO), Safe Abortion: Technical and Policy Guidance for Health Systems, second ed., Geneva: WHO, 2012.

2. Trussell J and Ellertson C, Estimating the efficacy of medical abortion, Contraception, 1999, 60(3):119-135.

3. Fjerstad $\mathrm{M}$ et al., Rates of serious infection after changes in regimens for medical abortion, New England Journal of Medicine, 2009. 361(2):145-151.

4. Shannon $C$ et al., Infection after medical abortion: a review of the literature, Contraception, 2004, 70(3):183-190.

5. Winikoff B et al., Safety, efficacy, and acceptability of medical abortion in China, Cuba, and India: a comparative trial of mifepristonemisoprostol versus surgical abortion, American Journal of Obstetrics \& Gynecology, 1997, 176(2):431-437.

6. Ngoc NTN et al., Comparing two early medical abortion regimens: mifepristone+misoprostol vs. misoprostol alone, Contraception, 2011, 83(5):410-417.

7. Henderson JT et al., Safety of mifepristone abortions in clinical use, Contraception, 2005, 72(3):175-178.

8. Gynuity Health Projects, Map of mifepristone approval, <http:// gynuity.org/resources/info/map-of-mifepristone-approval>, accessed July 10, 2012.

9. Rowlands S, Abortion pills: under whose control? Journal of Family Planning and Reproductive Health Care, 2012, 38(2):117-122.

10. Gynuity Health Projects, Map of misoprostol approval, <http:// gynuity.org/resources/info/map-of-misoprostol-approval>, accessed July 10, 2012.

11. Barbosa RM and Arilha M, The Brazilian experience with Cytotec, Studies in Family Planning, 1993, 24(4):236-240.

12. Costa SH and Vessey MP, Misoprostol and illegal abortion in Rio de Janeiro, Brazil, Lancet, 1993, 341(8855):1258-1261.

13. Billings DL, Misoprostol alone for early medical abortion in a Latin American clinic setting, Reproductive Health Matters, 2004, 12(24, Suppl.):57-64.

14. Sayette $\mathrm{H}$ et al., Buccal use of misoprostol alone for early abortion: the experience in four Latin American countries, Contraception,

\section{1, 84(3):304}

15. Borgatta L et al., Misoprostol as the primary agent for medical abortion in a low-income urban setting, Contraception, 2004, 70(2):121-126

16. von Hertzen $\mathrm{H}$ et al., Efficacy of two intervals and two routes of administration of misoprostol for termination of early pregnancy: a randomised controlled equivalence trial, Lancet, 2007, 369(9577): 1938-1946

17. Khan KS et al., WHO analysis of causes of maternal death: a systematic review, Lancet, 2006, 367(9516):1066-1074

18. Center for Reproductive Rights, The World's Abortion Laws Map 2007, Mar. 3, 2009, <http://reproductiverights.org/en/document/ the-worlds-abortion-laws-map-2007>, accessed Aug. 4, 2012

19. Swica Y et al., Review of the literature on patient satisfaction with early medical abortion using mifepristone and misoprostol, Expert Review of Obstetrics \& Gynecology, 2011, 6(4):451-468.

20. Fernandez MM et al., Assessing the global availability of misoprostol, International Journal of Gynaecology \& Obstetrics, 2009, 105(2): 180-186.

21. Dalvie S, Secretariat, Asia Safe Abortion Partnership, Mumbai, India, personal communication, July 2, 2012

22. Gomperts $\mathrm{R}$ et al., Regional differences in surgical intervention following medical termination of pregnancy provided by telemedicine, Acta Obstetricia et Gynecologica Scandinavica, 2012, 91(2): 226-231.

23. Gomperts RJ et al., Using telemedicine for termination of pregnancy with mifepristone and misoprostol in settings where there is no access to safe services, BJOG, 2008, 115(9):1171-1178.

24. Grossman D et al., Effectiveness and acceptability of medical abortion provided through telemedicine, Obstetrics \& Gynecology, 2011, 118(2 Pt. 1):296-303.

25. Warriner IK et al., Can midlevel health-care providers administer early medical abortion as safely and effectively as doctors? A randomised controlled equivalence trial in Nepal, Lancet, 2011, 377(9772):1155-1161.

26. Hajri $S$ et al., Ten years of medical abortion services and innovations in Tunisia, European Journal of Contraception \& Reproductive Health Care, 2008, 13(Suppl. 2):25-26.

27. Hajri S, Medical abortion: the Tunisian experience, African Journal of Reproductive Health, 2004, 8(1):63-69.

28. Yarnall J, Swica Y and Winikoff B, Non-physician clinicians can safely provide first trimester medical abortion, Reproductive Health Matters, 2009, 17(33):61-69.

29. Blum J et al., Can at-home semi-quantitative pregnancy tests serve as a replacement for clinical follow-up of medical abortion: a United States study, Contraception, 2012 (forthcoming).

30. Blum J, Gynuity Health Projects, New York, personal communication, Aug. 1, 2012

31. Dalvie S, Secretariat, Asia Safe Abortion Partnership, Mumbai, India, personal communication, Feb. 18, 2010.

32. Sanhueza P, Coordinación de Salud Sexual y Reproductiva, Secretaría de Salud del Distrito Federal, Ciudad de Mexico, personal communication, July 9, 2012.

33. Tsereteli T, Gynuity Health Projects, Tbilisi, Republic of Georgia, personal communication, Aug. 7, 2012.

Author contact: bwinikoff@gynuity.org 